\title{
New Parallel Interference Cancellation for Coded MC-DS-CDMA
}

\author{
Guoxiong $\mathrm{Xu}^{1}$ and Feng $\mathrm{Ji}^{2}$ \\ ${ }^{1}$ School of Electric and Information Engineer, Zhongyuan University of Technology, Zhengzhou, China \\ ${ }^{2}$ Library, Zhongyuan University of Technology, Zhengzhou, China
}

\begin{abstract}
In this paper, by the application of Turbo principle, a new parallel interference cancellation and decoding (PIC/decoding) algorithm for convolutionally-coded multi-carrier DS-CDMA (MC-DS-CDMA) is proposed. We give a detailed description of the proposed algorithm, and further mathematical analysis is also performed over it. The numerical simulation is made and corresponding results are obtained. From the numerical results we conclude that the proposed algorithm acquires much better performance than the conventional PIC/decoding, and what's more, if a posteriori log-likelihood rate (LLR) rather than a priori LLR is used to estimate the tentative values at each interference cancellation stage, the proposed algorithm exihibits more dominant performance advantages.
\end{abstract}

Keywords-multi-carrier; code division multi-access; loglikelihood rate (LLR); frequency diversity; BCJR algorithm

\section{INTRODUCTION}

In 1993, Berrou, et al creatively proposed a novel errorcorrecting code, known as Turbo codes [1], in which iterative decoding scheme is used. The iterative decoding is performed by soft-in and soft-out (SISO) recursion between component codes. After many enough iterations, Turbo code's performance has been proved to approach Shannon limit. Currently, the iterative idea is not only applied in the decoding of Turbo codes, but also has been extended in many aspects as an effective signal processing algorithm [2]. The extended iterative idea is called Turbo principle.

In general, CDMA systems consist of two parts, which are convolutional codes and CDMA, respectively. The two parts can be considered as the concatenation of block of error control codes with the CDMA channel block. In the concatenation system, Turbo principle can be put to good use and be expected to bring system performance improvement. In [3], Turbo-based CDMA iterative multi-user interference reduction (Turbo CDMA) was proposed and explored in detail.

$\operatorname{In}[4], X$. Wang, et al. proposed an iterative soft interference cancellation and decoding, in which the a posteriori probability CDMA decoder was replaced with a simple canceller and a single-user MMSE. However, in each iteration, soft interference cancellation and simultaneous MMSE are needed, and therefore there is a polynomial complexity in system implementation.

In this paper, we combine Turbo principle with convolutionally coded multi-carrier DS-CDMA (MC-DSCDMA), and propose a new parallel interference cancellation and decoding. The proposed PIC/decoding algorithm is performed soft interference cancellation and frequency diversity combining, respectively, to obtain decision statistics. With the approximate assumption of conditionally Gaussian distribution[5-6] taken, decision statistics for each coded symbol is converted into a priori log-likelihood rate (LLR), and input to the decoders. The proposed algorithm greatly lowers implementation complexity by replacing simultaneous MMSE interference suppression by frequency diversity combining for each iteration.

\section{SYSTEM DESCRIPTION}

\section{A. Transmitter Structure}

The transmitter structure for the discussed MC-DS-CDMA system is demonstrated as Figure I. Each information bit sequence is convolutionally encoded, and is then replicated into a number of identical sequences by $\mathrm{S} / \mathrm{P}$ converting. Each sequence is sent to a corresponding DS-CDMA modulator.

\section{B. Receiver Structure}

The proposed PIC/decoding structure for MC-DS-CDMA is illustrated in Figure II.

For convenience of description, in Figure II we only illustrate the $r$-th iteration process for user $k$. In each iteration, multi-access interference (MAI) is estimated and cancelled from the received signals.

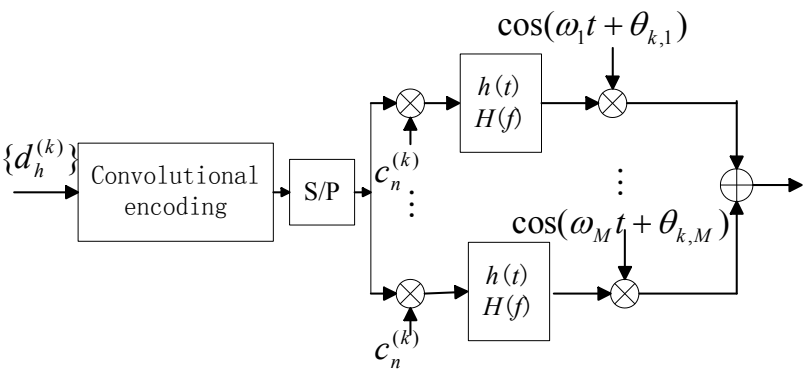

FIGURE I. THE TRANSMITTER STRUCTURE FOR MC-DS-CDMA WITH CONVOLUTIONAL ENCODING 


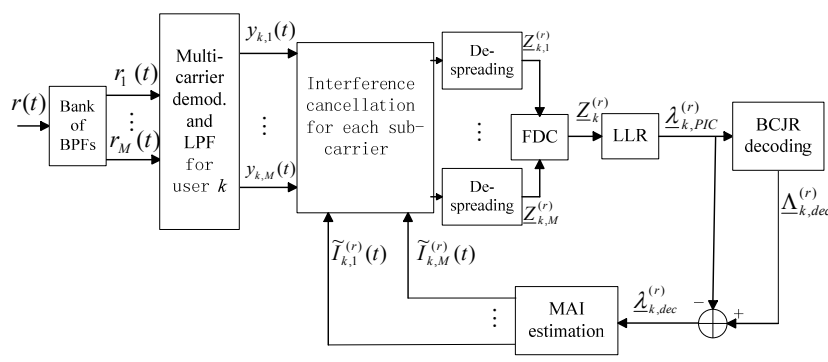

FIGURE II. THE PROPOSED PIC/DECODING STRUCTURE FOR MCDS-CDMA WITH CONVOLUTIONAL ENCODING

\section{NUMERICAL ANALYSIS}

A convolutional encoder with constraint length $v$ and rate $1 / n$ is considered for the proposed algorithm. Binary information bit $b_{k}(p)$ at the $p$-th bit interval for user $k$ is input to the corresponding convolutional encoder, the $q$-th coded symbol output is expressed as $d_{k, p}^{q},(q=1,2, \ldots, n)$, which can also be written as $d_{k}((p-1) n+q)$ in other form. Multicarrier modulation (MCM), spreading and multiplication are performed for each user's coded symbols .

Rayleigh fading channel is assumed, which complex transfer impulse response can be expressed as $\xi_{k, m}=\alpha_{k, m} \exp \left(j \beta_{k, m}\right)$, where $\left\{\alpha_{k, m}\right\}$ and $\left\{\beta_{k, m}\right\}$ are, respectively, an independently, identically distributed (i.i.d.)Rayleigh random variable with unit second moment and an i.i.d. uniform random variable over $[0,2 \pi)$. The received signal is expressed as

$$
r(t)=\sum_{k=1}^{K} \sqrt{2 E_{c}} \sum_{j=0}^{L-1} d_{k}(j) c_{k}(t-j T) \sum_{m=1}^{M} \alpha_{k, m} \cos \left(\omega_{m} t+\theta_{k, m}^{\prime}\right)+n(t)
$$

where $T$ is the duration of coded symbol, $\left\{\omega_{m}\right\}$ is sub-carrier frequency, $\left\{\theta_{k, m}\right\}$ are random variables with a uniform distribution over $[0,2 \pi), \theta_{k, m}^{\prime}=\theta_{k, m}+\beta_{k, m}, L$ is the frame length, and $n(t)$ is baseband additive white Gaussian noise (AWGN) with zero mean and two-sided power spectral density of $\eta_{0} / 2$, and

$$
c_{k}(t)=\sum_{l=-\infty}^{\infty} c_{k}(l) h\left(t-l T_{c}\right)
$$

where $c_{k}(l) \in\{-1,1\}$.

The $r(t)$ is band-pass filtered, demodulated and low-pass filtered (LPF), respectively, and we obtain demodulated signal for the $m$-th sub-carrier as

$$
\begin{aligned}
y_{k, m}(t)= & \sqrt{E_{c}} \sum_{k^{\prime}=1}^{K} \sum_{j=0}^{L-1} \alpha_{k^{\prime}, m} d_{k^{\prime}}(j) \sum_{n=0}^{N-1} c_{k^{\prime}}^{(j N+n)} x\left(t-j T-n T_{c}\right) \cos \left(\theta_{k^{\prime}, m}-\theta_{k, m}\right) \\
& +n_{k, m}(t)
\end{aligned}
$$

for the $m$-th sub-carrier, where $n_{m}(t)$ is the output from the $m$-th band-pass filter, corresoponding to $n(t)$.

The proposed PIC/decoding includes a few iterations, and each iteration is performed between the PIC and BCJR decoding. Here we only give the description of the $r$-th iteration without any loss of generality. For clarity, the PIC and BCJR decoding in the $r$-th iteration process are detailed in the following, respectively.

(1) PIC For PIC, interference cancellation (IC) for each subcarrier and frequency diversity combining (FDC) are performed and the a priori LLRs produced by PIC are computed.

Consider interference cancellation for the $i$-th coded symbol of user $k$. The external information $\left\{\underline{\lambda}_{k^{\prime}, d e c}^{(r-1)}, k^{\prime}=1,2, \cdots, K\right\}$ from the decoders in the $(r-1)$-th iteration is used as a priori information of PIC in the $r$-th iteration, where $\underline{\lambda}_{k^{\prime}, \text { dec }}^{(r-1)}=\left\{\lambda_{k^{\prime}, \text { dec }}^{(r-1)}\left(i^{\prime}\right), i^{\prime}=0,1, \cdots, L-1\right\}$. From [5], we obtain

$$
\widetilde{d}_{k^{\prime}}^{(r-1)}\left(i^{\prime}\right)=\tanh \left(\lambda_{k^{\prime}, \text { dec }}^{(r-1)}\left(i^{\prime}\right) / 2\right)
$$

where $\widetilde{d}_{k^{\prime}}^{(r-1)}\left(i^{\prime}\right)$ is soft value of coded symbol in terms of $a$ priori LLR, and is used to produce spreading signal by respreading. The $i$-th coded symbol is interfered by the other users' coded symbols with the same interval. Thus, the MAI for the $m$-th sub-carrier is estimated as

$$
\widetilde{I}_{k, m}^{(r)}(t)=\sqrt{E_{c}} \sum_{\substack{k^{\prime}=1 \\ k^{\prime} \neq k}}^{K} \widetilde{d}_{k}^{(r-1)}(i) c_{k^{\prime}}(t-i T) \cos \left(\theta_{k^{\prime}, m}^{\prime}-\theta_{k, m}^{\prime}\right)
$$

The $y_{k, m}^{(r)}(t)$ can be acquired by $y_{k, m}^{(r)}(t)=y_{k, m}(t)-\operatorname{LPF}\left\{\widetilde{I}_{k, m}^{(r)}(t)\right\}$ after $\widetilde{I}_{k, m}^{(r)}(t)$ is low-pass filtered and then subtracted from $y_{k, m}(t)$. The $y_{k, m}^{(r)}(t)$ is then despread, and the output for each sub-carrier $Z_{k, m}^{(r)}(i)(m=1,2, \ldots, M)$ can be obtained. $Z_{k, m}^{(r)}(i)$ is conditionally Gaussian distributed with mean $\sqrt{E_{c}} \alpha_{k, m} d_{k}(i)$ and variance computed as follows

$$
\begin{gathered}
\sigma_{k, m}^{(r)}=\operatorname{Var}\left[Z_{k, m}^{(r)}(i) \mid \alpha_{k, m}\right] \\
=\sum_{k^{\prime}=1, k^{\prime} \neq k}^{K} E_{c} \rho_{k^{\prime}, k}^{2} / 2 N^{2}-\sum_{k^{\prime}=1, k^{\prime} \neq k}^{K} E_{c}\left(\widetilde{d}_{k^{\prime}}^{(r-1)}(i) \rho_{k^{\prime}, k}\right)^{2} / 2 N^{2}+\eta_{0} / 2 N
\end{gathered}
$$

Frequency Diversity Combining (FDC) is applied to the $Z_{k, m}^{(r)}(i)(m=1,2, \ldots, M)$, and we obtain the decision statistics of coded symbol $d_{k}(i)$ in the $r$-th iteration 


$$
Z_{k}^{(r)}(i)=\sum_{m=1}^{M} g_{k, m}^{(r)} Z_{k, m}^{(r)}
$$

where $g_{k, m}^{(r)}$ is combining factor, which was discussed in [6]. In the context, we assume $g_{k, m}^{(r)}=\alpha_{k, m}$.

The $Z_{k}^{(r)}(i)$ is used to derive the a priori LLR of $d_{k}(i)$, and we obtain

$$
\lambda_{k, P C C}^{(r)}(i)=\log \frac{\mathrm{P}^{(r)}\left[d_{k}(i)=+1\right]}{\mathrm{P}^{(r)}\left[d_{k}(i)=-1\right]}=\log \frac{\mathrm{P}\left[Z_{k}^{(r)}(i) \mid d_{k}(i)=+1\right]}{\mathrm{P}\left[Z_{k}^{(r)}(i) \mid d_{k}(i)=-1\right]}
$$

The $Z_{k}^{(r)}(i)$ is conditionally Gaussian distributed with mean and variance computed as

$$
\begin{gathered}
\mathrm{E}\left[\mathrm{Z}_{\mathrm{k}}^{(\mathrm{r})}(i) \mid \alpha_{k, m}\right]=\sqrt{E_{c}} \sum_{m=1}^{M} g_{k, m}^{(r)} \alpha_{k, m} d_{k}(i)= \pm \sqrt{E_{c}} \sum_{m=1}^{M} g_{k, m}^{(r)} \alpha_{k, m} \\
\sigma_{k}^{(r)}=\operatorname{Var}\left[Z_{k}^{(r)}(i) \mid \alpha_{k, m}\right]=\sum_{m=1}^{M} g_{k, m}^{(r) 2} \operatorname{Var}\left[Z_{k, m}^{(r)}(i) \mid \alpha_{k, m}\right]
\end{gathered}
$$

Using (7) (9), we have

$$
\lambda_{k, P I C}^{(r)}(i)=\frac{2 \mathrm{E}\left[Z_{k}^{(r)}(i) d_{k}(i)\right]}{\left(\sigma_{k}^{(r)}\right)^{2}} Z_{k}^{(r)}(i)
$$

The a priori LLRs computed by (10)for the set of coded symbols are sent to the decoders for BCJR decoding.

(2) BCJR decoding Each decoder doesn't perform decoding for information bits until final iteration. Instead, it uses the $a$ priori LLRs given by PIC to updates a priori LLR for each coded symbol by BCJR algorithm[7]. In final iteration, the decoder makes the hard decision of information bits.

Consider the a priori LLR for the $i$-th coded symbol of user $k$. From (16) in [4], we get the a posteriori LLR of $d_{k}(i)$ for the $r$-th iteration

$$
\begin{gathered}
\Lambda_{k, \text { dec }}^{(r)}\left[d_{k}(i)=d_{k, t}^{j}\right]=\log \frac{\mathrm{P}^{(r)}\left[d_{t}^{j}=+1 \mid \text { decoding }\right]}{\mathrm{P}^{(r)}\left[d_{t}^{j}=-1 \mid \text { decoding }\right]} \\
=\log \frac{\sum_{s_{j}^{j}} \alpha_{t-1}^{(r)}\left(s^{\prime}\right) \beta_{t}^{(r)}(s) \coprod_{h=1}^{n} \mathrm{P}^{(r)}\left[d_{k, t}^{h}\left(s^{\prime}, s\right)\right]}{\sum_{s_{j}^{-}} \alpha_{t-1}^{(r)}\left(s^{\prime}\right) \beta_{t}^{(r)}(s) \coprod_{h=1}^{n} \mathrm{P}^{(r)}\left[d_{k, t}^{h}\left(s^{\prime}, s\right)\right]} \\
=\log \frac{\sum_{S_{j}^{+}} \alpha_{t-1}^{(r)}\left(s^{\prime}\right) \beta_{t}^{(r)}(s) \coprod_{h \neq j}^{n} \mathrm{P}^{(r)}\left[d_{k, t}^{h}\left(s^{\prime}, s\right)\right]}{\sum_{S_{j}^{j}} \alpha_{t-1}^{(r)}\left(s^{\prime}\right) \beta_{t}^{(r)}(s) \coprod_{h \neq j}^{n} \mathrm{P}^{(r)}\left[d_{k, t}^{h}\left(s^{\prime}, s\right)\right]}+\log \frac{\mathrm{P}^{(r)}\left[d_{k, t}^{j}\left(s^{\prime}, s\right)\right]}{\mathrm{P}^{(r)}\left[d_{k, t}^{j}\left(s^{\prime}, s\right)\right]} \\
=\lambda_{k, d e c}^{(r)}\left(d_{k, t}^{j}\right)+\lambda_{k, P I C}^{(r)}\left(d_{k, t}^{j}\right)
\end{gathered}
$$

The derivation process of (11) was detailed in [4].
From (11), $\quad \lambda_{k, P I C}^{(r)}\left(d_{k, t}^{j}\right)=\lambda_{k, P I C}^{(r)}\left(d_{k, t}^{j}\right)+\lambda_{k, d e c}^{(r)}\left(d_{k, t}^{j}\right)$, where $\lambda_{k, P I C}^{(r)}\left(d_{k, t}^{j}\right)$ is produced by PIC, and updated by the corresponding BCJR decoder to obtain $\lambda_{k, d e c}^{(r)}\left(d_{k, t}^{j}\right)$, which is then fedback to PIC as a priori LLR in subsequent iteration.

In the iteration process, initial a priori LLR input to PIC is set zero. The a posteriori LLRs from the decoder are expressed as

$$
\Lambda_{k, \text { dec }}^{(R)}\left[b_{k}(t)\right]=\log \frac{\sum_{U^{+}} \alpha_{t-1}^{(R)}\left(s^{\prime}\right) \beta_{t}^{(R)}(s) \coprod_{h=1}^{n} \mathrm{P}^{(R)}\left[d_{k, t}^{h}\left(s^{\prime}, s\right)\right]}{\sum_{U^{-}} \alpha_{t-1}^{(R)}\left(s^{\prime}\right) \beta_{t}^{(R)}(s) \coprod_{h=1}^{n} \mathrm{P}^{(R)}\left[d_{k, t}^{h}\left(s^{\prime}, s\right)\right]}
$$

for final iteration, where $R$ is the iteration number.

Finally, $b_{k}(t)$ is obtained by $\hat{b}_{k}(t)=\operatorname{sgn}\left(\Lambda_{k, d e c}^{(R)}\left(b_{k}(t)\right)\right)$.

\section{NUMERICAL RESULTS}

In order to evaluate the performance of the proposed $\mathrm{PIC} /$ decoding, in this section, we give numerical results by the computer simulation according to the above analysis. In the simulations, we choose simulation parameters as: (1)the user number $K=7$; (2) convolutional code with rate $1 / 2$,constraint length $v=3$ and generator matrix [1 111,101$]$; (3) randomly generated code sequences with spreading factor $N=11$; (4)subcarrier number $M=2$.

For the simulations, two cases are considered. One is that the a posteriori LLR produced by BCJR decoding is directly fed to the PIC, the other is a priori LLR is fed to the PIC for each iteration. For convenience of description, the former is called PIC/decoding with a posteriori LLR feedback and the latter is called PIC/decoding with a priori LLR feedback.

Figure III gives the performance of the proposed PIC/decoding with a priori LLR feedback, while Figure IV shows the performance with a posteriori LLR feedback, both for the first 3 iterations. They are both compared to the conventional receiver with no iteration in performance. It is obviously seen from both Figure III and Figure IV that the proposed PIC/decoding, whether with a priori LLR feedback or with a posteriori LLR feedback, outperforms conventional receiver with no iterations in performance. On the other hand, with the increase in iteration number, the bit error rate (BER) lowers, and system performance improves dominantly. For example, in Figure IV, at signal-noise-rate (SNR) of 8dB, the bit error rate (BER) for the proposed $\mathrm{PIC} /$ decoding with $a$ posteriori LLR feedback is about, $7 \times 10^{-3}, 2 \times 10^{-4}, 1 \times 10^{-4}$ after the first, second, third respectively, which is far lower than that of conventional receiver with no iterations, which is about $8 \times 10^{-2}$. It is also shown that the first two iterations lead to great performance improvement, but up to the third iteration, the system performance improves slightly. 


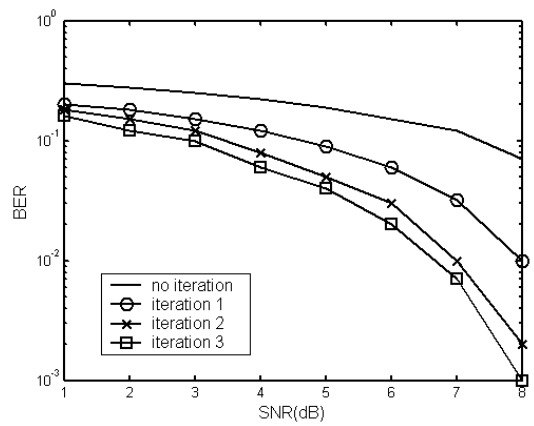

FIGURE III. PERFORMANCE OF THE PROPOSED PIC/DECODING WITH A PRIORI LLR FEEDBACK

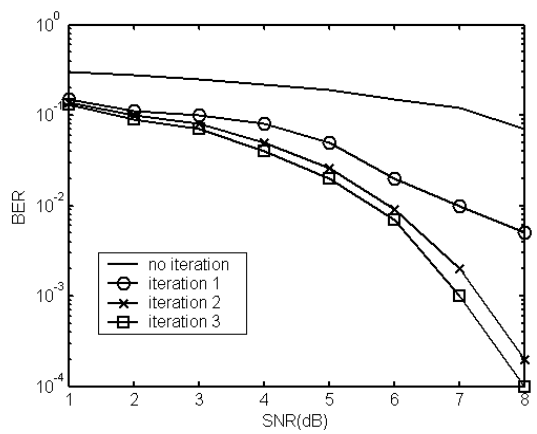

FIGURE IV. PERFORMANCE OF THE PROPOSED PIC/DECODING WITH A POSTERIORI LLR FEEDBACK

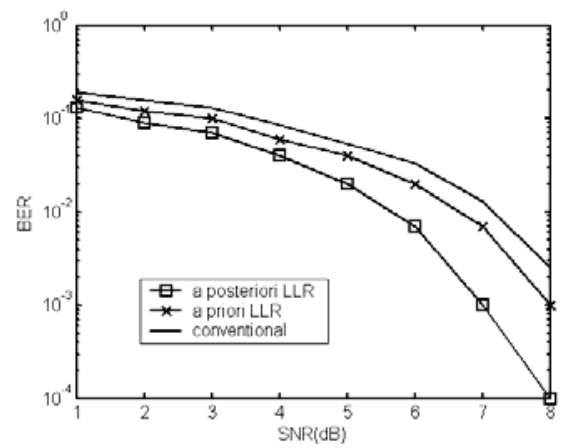

FIGURE V. PERFORMANCE COMPARISON OF THE PROPOSED PIC/DECODING AND CONVENTIONAL PIC/DECODING WITH FOUR ITERATIONS

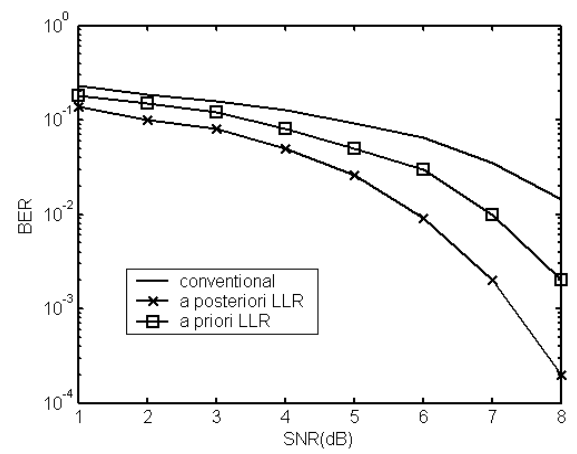

FIGURE VI. PERFORMANCE COMPARISON OF THE PROPOSED PIC/DECODING AND CONVENTIONAL PIC/DECODING WITH FOUR ITERATIONS
In Figure $\mathrm{V}$ and Figure $\mathrm{VI}$, the performance of the proposed PIC/decoding after two and three iterations is shown, respectively, and compared to conventional PIC/decoding. It is seen that the proposed PIC/decoding with a posteriori LLR feedback shows the best performance, while the proposed PIC/decoding with a priori LLR feedback is dominantly inferior to that with a posteriori LLR feedback. For example, for three iterations (as shown in Figure VI), at the SNR of $8 \mathrm{~dB}$, the BER for the proposed PIC/decoding with a posteriori LLR feedback is about $10^{-4}$, while BER for that with a priori LLR feedback is about $10^{-3}$. It is also noticed that the proposed $\mathrm{PIC} /$ decoding, whether with a posteriori LLR feedback or with a priori LLR feedback, outperforms the conventional $\mathrm{PIC/decoding} \mathrm{in} \mathrm{performance} \mathrm{significantly.}$

\section{CONCLUSIONS}

Applying Turbo principle to convolutionally coded multicarrier DS-CDMA, we proposed a new PIC/decoding algorithm. The theoretical analysis and numerical simulation were made, respectively.

It is shown that the proposed PIC/decoding obtains the most dominant performance improvement for the first two iterations, but up to the third iteration, the performance improves slightly. It is also shown that the proposed PIC /decoding with $a$ posteriori LLR feedback always outperforms that with a priori LLR feedback in performance. The proposed PIC/decoding, whether with a posteriori LLR feedback or with a priori LLR feedback, outperforms the conventional PIC/decoding in performance significantly. It should be noticed that compared to a priori LLR feedback, the proposed PIC/decoding with $a$ posteriori LLR feedback has a lower implementation complexity, since for the latter, a posteriori LLR produced by the decoders is directly fedback to PIC, while for the former, the a priori LLR must be separated from a posteriori LLR before feedback to PIC, which increase the implementation complexity.

\section{REFERENCES}

[1] C.Berrou, A. Galvieux, and P. Thitimajshima., "Near Shannon limit error-correcting coding and decoding: Turbo codes," In proc. ICC'93, Geneva, Switzerland, vol.1, pp.1064-1070, May 1993.

[2] J.Hagenauer, "The Turbo principle: Tutorial introduction and state of the art," In proc. International Symposium on Turbo codes and Related Topics, Brest, France, pp.1-11, Sept.1997.

[3] P.D.Alexander, M.C.Reed, J.A.Asenstorfer, and C.B.Schlegel, "Iterative multiuser interference reduction: Turbo CDMA," IEEE Trans. Commun., vol.47, No.7, pp.1008-1014, July 1999.

[4] X. Wang and H. Vincent, "Iterative (Turbo) soft interference cancellation and decoding for coded CDMA," IEEE Trans. Commun., vol.47, pp.1046-1061, July 1999.

[5] L. C. Gan and G.X.Xu, "Turbo interference cancellation/decoding for cnvolutionally coded multi-carrier DS-CDMA," Chinese Journal of Electronics, vol.14,pp.309-313, April 2005

[6] G.X.Xu, "Turbo IC/decoding for multi-carrier DS-CDMA," in Proc.of ICCC2013, Wuhan, pp.74-77, Nov.2013.

[7] L. R. Bahl, J. Cocke, F. Jelinek, and J. Raviv, "Optimal decoding of linear codes for minimizing symbols error rate," IEEE Trans. Inform. Theory, Vol. IT-20, No.3, pp.284-287, Mar.1974. 